\title{
SYNTACTIC AND LEXICAL COMPLEXITY OF TURKISH UNIVERSITY STUDENTS' SPOKEN ENGLISH DISCOURSES
}

\author{
Ozan Kaya ${ }^{1 *}$, Hatice Gülru Yüksel ${ }^{2}$ \\ ${ }^{1}$ Foreign Languages Education Department (Yıldız Technical University), Turkey, \\ ozankayaytu@gmail.com \\ ${ }^{2}$ Prof. Dr., Foreign Languages Education Department (Yıldız Technical University), Turkey, \\ gulruyuksel2002@gmail.com \\ ${ }^{*}$ Corresponding Author
}

\begin{abstract}
English language has become an international language and many academics and researchers have been working to find a more efficient and convenient approach to teach and test it. Especially, speaking is one of the skills that English as a Foreign Language students need to master and is regarded as one of the most difficult skills in the learning experience. Speaking skill is especially crucial for university students as it is vital for academic performance, for graduating from university, for pursuing a good career and successful communication. This study aims to investigate the syntactic and lexical complexity of the B1 level of English learners' spoken discourse, as well as examining the effect of speaking task type on syntactic and lexical complexity. The study was conducted with 30 Turkish university students. Three different speaking tasks were employed: a personal task, a descriptive task, and a narrative task. Students' spoken productions were transcribed and examined utilizing web-based syntactic and lexical complexity analyzers. The numerical data obtained from the analyzers were analyzed in the SPSS program. The findings showed that there was no statistically significant correlation between the syntactic and lexical complexity development of the students. The results indicated that the task type had an effect on the syntactic and lexical complexity in spoken discourse. The most syntactically and lexically complex sentences were produced in the personal task and the second-best complexity was measured in the descriptive task. This study has ramifications for material designers, EFL instructors, and test developers. The current study's findings revealed that subject familiarity is indeed very important in spoken production. Topic and subject familiarity should be taken into account while designing speaking tasks for lower proficiency levels.
\end{abstract}

Keywords: Syntactic Complexity, Lexical Complexity, Spoken Discourse.

\section{INTRODUCTION}

Speaking is one of the skills that English as a Foreign Language (Henceforth EFL) students need to master and is regarded as one of the most difficult skills in the learning experience. Speaking together with writing is seen as a productive skill, whereas listening and reading are the receptive skills. The students can communicate in the form of a speech in order to give the listener an idea or the message. It is also necessary in the speaking process to have a solid speech structure so that the listeners can get the information or ideas from the speakers. Speaking skill is especially crucial for university students as it is vital for academic performance, for graduating from university, for pursuing a good career and successful 
communication. In SLA (Henceforth Second Language Acquisition) studies, it has been observed that writing has been given more importance than speaking while speaking discourse is mostly neglected by the researchers in the field of linguistics. A short history of linguistic pedagogical approach which has seen different methods and techniques all through the SLA indicates a step towards oral mastery instead of reading understanding as a purpose for studying languages (Richards, Rodgers, 2001). Therefore, speaking has become even more important in terms of language acquisition and proficiency. Most notably, learner proficiency in a language is generally measured by testing the student's performance and the production that learners produce in the target language. It is widely known that the language produced by the learners is a useful 2 source of SLA forms since the sentences that learners create encourage them to work deeply with language and mentally than the receptive skills (Skehan, 1998). These productions are both written or spoken. Furthermore, academics and instructors have taken a task as a study instrument to develop SLA to gain awareness of the underlying reasons of second language acquisition to advance in instructional practices. In the SLA research, tasks were frequently employed as a tool for the generation of language creation, communication, content interpretation, information analysis and structure emphasis that are intended to encourage the learning of a second language (Henceforth L2) (Van den Branden, 2006). Tasks in relation with everyday life could be structured in order to promote their significance and conversational character since there is certainly no question that these tasks contribute to the creation of a feasible way for learning, frequently combining over one competence at the same time (Skehan, 1996). In addition, assessment of these productive skills may be achieved in line with the aspects of productive skills in a task characterized by a focus on various linguistic systems, such as complexity, accuracy and fluency (Skehan, 1998). The three aspects of tasks contain measurements that are relevant to distinct linguistic features. Among these three aspects, complexity which is the main focus of this thesis is mostly neglected in SLA research.

The complexity among the complexity, accuracy and fluency (Henceforth CAF) triad is undoubtedly the most difficult structure due to its multiple meanings. Initially, the phrase complexity is employed to relate to tasks and linguistic performance. It has several interpretations although it limits the usage of complexity to performing definitions since it may be used in other areas of language and conversation (Pallotti, 2009). Moreover, complexity includes two subheadings, such as syntactic and lexical complexity which will be the main focus of this thesis.

In addition, the discipline of SLA is less interested in assessing the spoken production of the students than writing as a unit on the basis of task performance. As a result, this research may aid in a deeper understanding of how different task types impact the students' selection of language forms/ lexical items and the complexity aspects regarding the task types. As a result, the findings of this research will be useful in teaching speaking skills. Moreover, the results of the research will be useful to EFL instructors, material developers and testing who would like to use speaking tasks to improve spoken production of their students and processes to teach. Practically, this research is supposed to present a profound vision for the English language educators about syntactic and lexical complexity, these terms' descriptions and testing these features for their students by being aware of the importance of the issue. The research might potentially provide empirical evidence for the relationship between syntactic and lexical complexity in spoken discourse. This research investigated the effect of speaking task types and syntactic and lexical complexity of the spoken discourse of the students and also the relationship between syntactic and lexical complexity. In this sense, our research has the potential to add to the current literature in a variety of ways.

\subsection{Research Questions}

This study aims to answer the following research question:

1) What is the syntactic and lexical complexity level of B1 level Turkish L2 learners?

2) What is the relationship between syntactic and lexical complexity in B1 level Turkish L2 learners' spoken performance?

\subsection{Literature Review}

This chapter gives an overview of research which emphasizes the influence of task complexity on linguistic production, especially regarding speaking, in accordance with the goal of the current research. On storytelling tasks that imitated the capacity to narrate situations, the research investigated variations in the creation of spoken storytelling in 12 grownup L2 students. Syntactic and lexical complexity, as well as MLU, were assessed. The findings backed with the theory that complicated tasks generate more precise and complicated production than easier tasks (Robinson, 1995). Student judgments of task difficulty and the 
connections with four being fluent metrics under three various tasks, unlinked image descriptions, narrative, and linked cartoons, were investigated from the point of view of English as a Second Language (Henceforth ESL) French students. Even though the students' judgments of vocabulary and being fluent varied in every task, these factors appeared to be useful in determining the total task difficulty. One interesting finding was that students' being fluent differed between the three tasks regarding articulated words' ratio and mean hesitation duration despite not regarding hesitation recurrence or articulation ratio (Préfontaine \&Kormos, 2015).

An image sequence was incorporated in a traditional storytelling task that consists of a sequence of ordered images. The complexity levels for this image series were substantially greater. This result has been confirmed by other researchers, who found that the ability to connect image parts is a reliable means of boosting linguistic complexity. The degree of linguistic complexity appropriate for a given task can be influenced by task design (Tavakoli \& Skehan, 2005).

A deep investigation of spoken skills on two various tasks - making a presentation and debate - was conducted utilizing objective criteria including such production and complexity metrics to analyse the influence between structural complexity indicators, the kind of task, and diagnostic evaluation of students' oral competency. In terms of the impact of the kind of task on spoken discourse complexity, the findings revealed that while there was a stronger correlation between all four production aspects and the presentation tasks' results. Namely, students generated more complicated spoken performances regarding linguistic production compared to the debate. It was mentioned that in both tasks, the mean length of units (Henceforth MLU) appeared to be a more trustworthy metric for estimating students' spoken linguistic skills (Gan, 2012).

In the spoken competence examinations of 107 learners of EFL in Tianjin, China, the connection between holistically judgements of spoken competency and objective criteria of syntactic development is investigated. For three various interview tasks (Description/Narrative, Role - play, and Asking a Question), the research analyzes a holistic score of level of achievement with objective criteria including such Mean T-Unit Length. In Halleck's study, the intermediate students $(N=10)$ achieved a score of 8.02 in narrative and description tasks, and they achieved a score of 6.11 in role-play and asking a question tasks for Mean T-Unit Length. This research demonstrates that objective criteria of syntactic development differ dramatically for participants evaluated at two of the three competence levels investigated, and that observed degrees of syntactic development change depending on the task (Halleck, 1995).

The production and syntactic complexity of school-aged children were evaluated in connection to their knowledge of the issue of debate, chess. 32 chess-playing youngsters consented to be questioned by an experienced evaluator with no chess expertise. Every kid's chess expertise was evaluated, and he or she was classed as a rookie or master player. General and chess talk, and chess explanation were the three spoken tasks that every youngster completed. Conversations were taped, typed into transcripts. They were segmented into T-units, and finite sentences were tagged. T-units, MLU, and clausal density usage were all examined within every oral task. In the chess explaining, T-units, MLU, clausal density and subordinate clauses were significantly greater than in the chess or general talk tasks. Masters understood better about chess, had competed for longer periods, and were superior competitors than rookies. The spoken task has a significant impact on overall linguistic production and syntactic complexity in school-aged children (Nippold, 2009).

The purpose of this research is to look into the impact of task type and competency degree on several elements of lexical complexity in spoken discourse monologues generated by intermediate and advanced students from Iran. Three separate spoken tasks were completed by 35 intermediate and advanced students from Iran. These tasks include an argumentative, a descriptive, and a narrative task. Three elements of lexical complexity were identified in the spoken monologues. These are diversity, density, and sophistication. The kind of task and competency degree had a substantial impact on lexical complexity in the students' task completion according to the findings. The argumentative task received the greatest marks for diversity and density whereas the narrative task received the best mark for sophistication (Bayazidi et al, 2020).

The similar methodological approach was employed to study the impact of three distinct kinds of speaking tasks (personal, storytelling, and decision-making) on two elements of lexical complexity which are lexical diversity and sophistication. The findings showed that storytelling tasks constantly generate the greatest levels of complexity. The personal tasks were not consistent in terms of lexical diversity; however the findings for the storytelling and decision-making tasks are the opposite of that for lexical sophistication. The narrative tasks produced statistically lower diversity than the decision-making tasks (Foster, 2001). 


\section{METHODOLOGY}

\subsection{Research Context and Participants}

The research was carried out in the preparatory school of a private university in Istanbul, Turkey where the researcher works as an English instructor in the second term of the 2020-2021 academic year. The preparatory school provides students to take courses at various language proficiency levels. The preparatory program has a modular system, with each level lasting eight weeks. All the students started to learn English at Elementary level with the placement test of the preparatory program. They are assigned to the proper classes based on their abilities. The students studied 24 hours of instruction per week in the preparatory program. All students took a Language Achievement Test (LAT) relevant to their level at the conclusion of each 8-week to decide whether they will pass or fail the module. In this study, they are expected to complete spoken assignments like talking about their own life or preferences, a description of a picture and storytelling and be evaluated on them. The students in this research are at intermediate level. Furthermore, the students took the online placement test of International House (www.testmylevel.com) and it was found out that they were at Intermediate level.

Table 1. Socio-Demographic Information of the Students

\begin{tabular}{|c|c|c|c|}
\hline & & Frequency & Percent \\
\hline Gender & Male & 12 & 40 \\
& Female & 18 & 60 \\
\hline Age & 18 & 8 & 27 \\
& 19 & 12 & 40 \\
& 20 & 6 & 20 \\
& $21-23$ & 4 & 13 \\
\hline Starting Age & $5-9$ & 3 & 10 \\
for English & 10 & 7 & 23,33 \\
& 11 & 13 & 43,33 \\
& $12-16$ & 7 & 23,33 \\
\hline 2nd Language & Yes & 1 & 3 \\
& No & 29 & 97 \\
\hline Total & & 30 & 100 \\
\hline
\end{tabular}

\subsection{Data Collection Procedure}

The following instruments were employed to conduct the research: three different speaking tasks (a personal task (personal tasks are easy tasks since the participants already know the information and this can lower the degree of complexity of the tasks), a picture description task and a narrative task (Although it offers some images, it can be difficult to organize the content for storytelling). A range of tasks were used in this study to see if different task types had an influence on EFL students' speaking performance.

Firstly, the students were informed about the research study and 30 students sent an email to the researcher stating that they would like to participate. Afterwards, the researcher convened a meeting with all of the students to explain the significance and the procedure of the study. The researcher responded to the student questions. The researcher described the tasks that would be included in the study. The participants were provided all of the essential instructions for completing the tasks. They were notified that they would be recorded during the tasks. All of this information was provided in Turkish. After being informed, the students were e-mailed a google form to obtain their consent to participate in the whole process. They were informed that they could withdraw from the study at any time they would like. They were told that their names would be anonymous throughout the whole study. In the following days, the researcher prepared a schedule to meet with the students for the speaking tasks. The researcher sent emails to the students about the meeting. The meetings were held on the Zoom platform. All the meetings were made in the same week. The researcher prepared a presentation slide to project the tasks on Zoom via share screen. The students read the questions and responded when they were ready. There was no time limitation. The duration of every task depended on the performance of the student. Before they started responding, they were given adequate time to look at the photographs and formulate their ideas. The researcher remained silent and did not ask any questions during the whole session not to affect the performance of the student. The researcher moved on to the next task when the students indicated they were finished speaking. The sessions were recorded on Zoom. The whole process was carried out only on the computers at home. The audios were transcribed into 
the Otter.ai transcription program. Each automated transcription was reviewed three times by the researcher to correct the transcription mistakes.

\subsection{Data Analysis}

In line with the literature, it was decided to analyze the spoken data for 12 main indices of syntactic complexity and 5 main indices of lexical complexity. Various automated tools for syntactic and lexical complexity were used because they appeared to make the analysis less burdensome, more practical and more unbiased (Crossley \& McNamara, 2009). Lu's (2010, 2012) web-based L2 Syntactic and Lexical Complexity Analyzer, 'Web-based L2SCA/LCA: Single Mode' was used to evaluate the syntactic and lexical complexity. Finally, the SPSS program was used to analyze the data gathered from Lu's (2010) web-based L2 Syntactic and Lexical Complexity Analyzer. The fact that all of the automated tools employed in this research are free and simple to employ is the most remarkable feature.

Table 2. Research Questions and the Types of Analysis

\begin{tabular}{|l|l|}
\hline Research Questions & Analysis \\
\hline $\begin{array}{l}\text { 1- What is the syntactic and lexical complexity level of B1 level } \\
\text { Turkish L2 learners? }\end{array}$ & Descriptive Statistics \\
\hline $\begin{array}{l}\text { 2- What is the relationship between syntactic and lexical } \\
\text { complexity in B1 level Turkish L2 learners' spoken performance? }\end{array}$ & Correlation Analysis \\
\hline
\end{tabular}

\section{RESULTS}

\subsection{Descriptive Statistics of Syntactic Complexity}

Descriptive statistics present the syntactic complexity level of the students. The syntactic complexity consists of two distinct indices such as syntactic structures (Word count (W), Sentence (S), Clause (C), T-unit (T), Dependent Clause (DC), Complex T-unit (CT and syntactic complexity indices (Mean length of sentence (MLS), Mean length of T-unit (MLT), Mean length of clause (MLC), Clause per sentence (C/S), T-unit per sentence (T/S), Complex T-unit ratio (CT/T). Descriptive statistics present the means (M) and standard deviations (SD) for the indices of syntactic complexity.

Table 3. Means and Standard Deviations of Syntactic Structures and Syntactic Complexity Indices of All Tasks

\begin{tabular}{|l|c|c|c|c|c|c|c|l|}
\hline & \multicolumn{2}{|c|}{ T1 } & T2 & & T3 & & AT & \\
\hline & $M$ & $S D$ & $M$ & $S D$ & $M$ & $S D$ & $M$ & $S D$ \\
\hline W & 118,56 & 43,66 & 101,76 & 46,81 & 100,93 & 37,78 & 321,26 & 107,10 \\
\hline S & 9,83 & 4,18 & 10,36 & 4,55 & 8,80 & 3,70 & 29,00 & 10,03 \\
\hline C & 19,30 & 7,28 & 18,00 & 7,13 & 15,93 & 6,38 & 53,23 & 16,89 \\
\hline T & 12,50 & 5,71 & 13,06 & 4,98 & 12,13 & 4,78 & 37,70 & 12,10 \\
\hline DC & 5,86 & 3,28 & 4,40 & 3,01 & 3,33 & 2,00 & 13,60 & 6,20 \\
\hline CT & 4,50 & 2,22 & 3,66 & 2,45 & 3,23 & 2,01 & 11,40 & 4,36 \\
\hline MLS & 12,56 & 2,83 & 10,01 & 2,35 & 11,94 & 3,25 & 11,17 & 1,53 \\
\hline MLT & 10,07 & 2,55 & 7,73 & 1,45 & 8,46 & 1,50 & 8,55 & 1,06 \\
\hline MLC & 6,19 &, 76 & 5,57 &, 81 & 6,46 & 1,05 & 6,03 &, 57 \\
\hline C/S & 2,03 &, 44 & 1,81 &, 45 & 1,85 &, 43 & 1,85 &, 25 \\
\hline T/S & 1,25 &, 14 & 1,29 &, 18 & 1,40 &, 24 & 1,30 &, 11 \\
\hline CT/T &, 38 &, 16 &, 28 &, 18 &, 26 &, 14 &, 30 &, 09 \\
\hline
\end{tabular}

As shown in Table 3 , task 1 has the highest mean $(M=118,56, S D=43,66)$ while task 3 has the lowest mean $(M=100,93, S D=37,78)$ in terms of word count. The students could produce more word in task 1 than task 2 and 3 . This shows that a personal task helps the students use more vocabulary while speaking. As for sentence, the students produced more sentences in description task than in other tasks. Task 2 has the highest mean $(M=10,36, S D=4,55)$. Task 3 has the lowest mean $(M=8,80, S D=3,70)$. However, when mean length of sentences is checked, it can be understood that shorter sentences were formed in Task 2. The longest sentences were formed in Task 1 and it also shows that the students are more successful at forming longer sentences in a personal task rather than descriptive or narrative tasks. As for 
clause, task 1 has the highest mean $(M=19,30, S D=7,28)$ while task 2 has the mean $(M=18,00, S D=$ $7,13)$ and task $3(M=15,93, S D=6,38)$. The students produced more clauses in task 1 than the other tasks. Although the means of T-unit are close to each other, the highest mean of T-unit belongs to Task 2 ( $M$ $=13,06, S D=4,98)$. When it comes to dependent clause, the students produced the highest mean $(M=$ $5,86, S D=3,28)$ in task 1 again. Moreover, task 1 has the highest complex T-unit mean $(M=4,50, S D=$ $2,22)$ while task 3 has the lowest mean $(M=3,23, S D=2,01)$. In terms of mean length of sentence, task 1 has the highest mean $(M=12,56, S D=2,83)$ while task 2 has the lowest mean $(M=10,01, S D=2,35)$. This shows that the students produced shorter sentences than the other tasks. In accordance with MLS, task 1 has the highest mean $(M=10,07, S D=2,55)$ while task 2 has the lowest mean $(M=7,73, S D=1,45)$. Surprisingly, task 3 has the highest mean $(M=6,46, S D=1,05)$ in terms of mean length of clause. In clause per sentence, task 1 has the highest mean $(M=2.03, S D=, 44)$ as well while task 2 has the lowest mean $(M$ $=1,81, S D=, 45)$. Interestingly, in terms of T-unit per sentence, task 3 has the highest mean $(M=1,40, S D=$ ,24) while task 1 has the lowest mean $(M=1,25 S D=, 14)$. In complex $T$-unit ratio, task 1 has the highest mean $(M=, 38, S D=, 16)$ whereas task 3 has the lowest mean $(M=, 26, S D=, 14)$.

\subsection{Descriptive Statistics for Lexical Complexity}

Descriptive statistics present the syntactic complexity level of the students. The lexical complexity consists of lexical density (LD), lexical sophistication-I (LS-1), number of different words (NDW), type-token ratio (TTR), and lexical word variation (LV).

Table 4. Means and Standard Deviations of Lexical Complexity Indices of All Tasks

\begin{tabular}{|l|l|l|l|l|l|l|l|l|}
\hline & \multicolumn{2}{|c|}{ T1 } & \multicolumn{2}{c|}{ T2 } & \multicolumn{2}{c|}{ T3 } & \multicolumn{2}{c|}{ AT } \\
\hline & $M$ & $S D$ & $M$ & $S D$ & $M$ & $S D$ & $M$ & $S D$ \\
\hline LD &, 50 &, 03 &, 45 &, 05 &, 48 &, 05 &, 47 &, 03 \\
\hline LS-1 &, 24 &, 07 &, 19 &, 06 &, 17 &, 08 &, 20 &, 03 \\
\hline NDW & 57,26 & 15,29 & 50,70 & 17,30 & 50,36 & 14,26 & 119,53 & 28,79 \\
\hline TTR &, 50 &, 07 &, 51 &, 07 &, 51 &, 07 &, 38 &, 05 \\
\hline LV &, 62 &, 09 &, 69 &, 10 &, 66 &, 08 &, 56 &, 08 \\
\hline
\end{tabular}

As shown in Table 4, task 1 has the highest mean $(M=, 50, S D=, 03)$ in lexical density while task 2 has the lowest mean $(M=, 45, S D=, 05)$. These statistics show that the students used more lexical words than content words in task 1 than the other tasks. In lexical sophistication, the students used the most sophisticated vocabulary in task $1(M=, 24, S D=, 07)$. They used the second most sophisticated words in task $2(M=, 19, S D=, 06)$ and the least one in task $3(M=, 17, S D=, 08)$. In terms of lexical variation, task 1 has the highest mean $(M=57,26, S D=15,29)$ in the number of different words. Task $2(M=50,70 S D=$ $17,30)$ and Task $3(M=50,36, S D=14,26)$ have really close means and standard deviations. Nonetheless, the students produced slightly higher mean in task 2 rather than task 3 . As for Type-Token Ratio, all tasks have quite close means $(M=, 50,51,51, S D=, 07,07,07)$, respectively task $1,2,3$. Finally, in lexical word variation index, task 1 has the lowest mean $(M=, 62, S D=, 09)$ while task 2 has the highest $(M=, 69, S D=$ ,10).

\subsection{Correlation Analysis between Syntactic and Lexical Complexity}

Table 5. The Relationship between Indices of Syntactic and Lexical Complexity of All Tasks

\begin{tabular}{|c|c|c|c|c|c|c|c|}
\hline & & & ATLD & ATLS-1 & ATNDW & ATTTR & ATLV \\
\hline \multirow{9}{*}{$\begin{array}{l}\text { Spearman's } \\
\text { Rho }\end{array}$} & \multirow[t]{2}{*}{ ATW } & \multirow{2}{*}{$\begin{array}{l}\text { Correlation Coefficient } \\
\text { Sig. (2-tailed) }\end{array}$} & ,212 &,- 148 &, 908 &,$- 695^{\star \prime \prime}$ & ,486" \\
\hline & & & ,262 & ,435 &, 000 & ,000 & ,006 \\
\hline & \multirow[t]{2}{*}{ ATS } & \multirow{2}{*}{$\begin{array}{c}\text { Correlation Coefficient } \\
\text { Sig. (2-tailed) }\end{array}$} & ,311 &,- 165 &, 862 &,- 626 &,- 422 \\
\hline & & & ,094 & ,385 &, 000 &, 000 &, 020 \\
\hline & \multirow[t]{2}{*}{ ATC } & \multirow{2}{*}{$\begin{array}{c}\text { Correlation Coefficient } \\
\text { Sig. (2-tailed) }\end{array}$} & ,210 &,- 288 &, 779 &,- 785 & 613 \\
\hline & & & ,265 & 123 &, 000 &, 000 &, 000 \\
\hline & \multirow[t]{2}{*}{ ATT } & \multirow{2}{*}{$\begin{array}{c}\text { Correlation Coefficient } \\
\text { Sig. (2-tailed) }\end{array}$} & ,237 &,- 197 & 810 &,- 689 &,- 459 \\
\hline & & & ,207 & ,297 &, 000 &, 000 &, 011 \\
\hline & ATDC & Correlation Coefficient &, 137 &,$- 443^{\star}$ &, 616 &,- 619 &, 527 \\
\hline
\end{tabular}


Proceedings of INTCESS 2022- 9th International Conference on Education \& Education of Social Sciences 17-18 January 2022- Online Conference

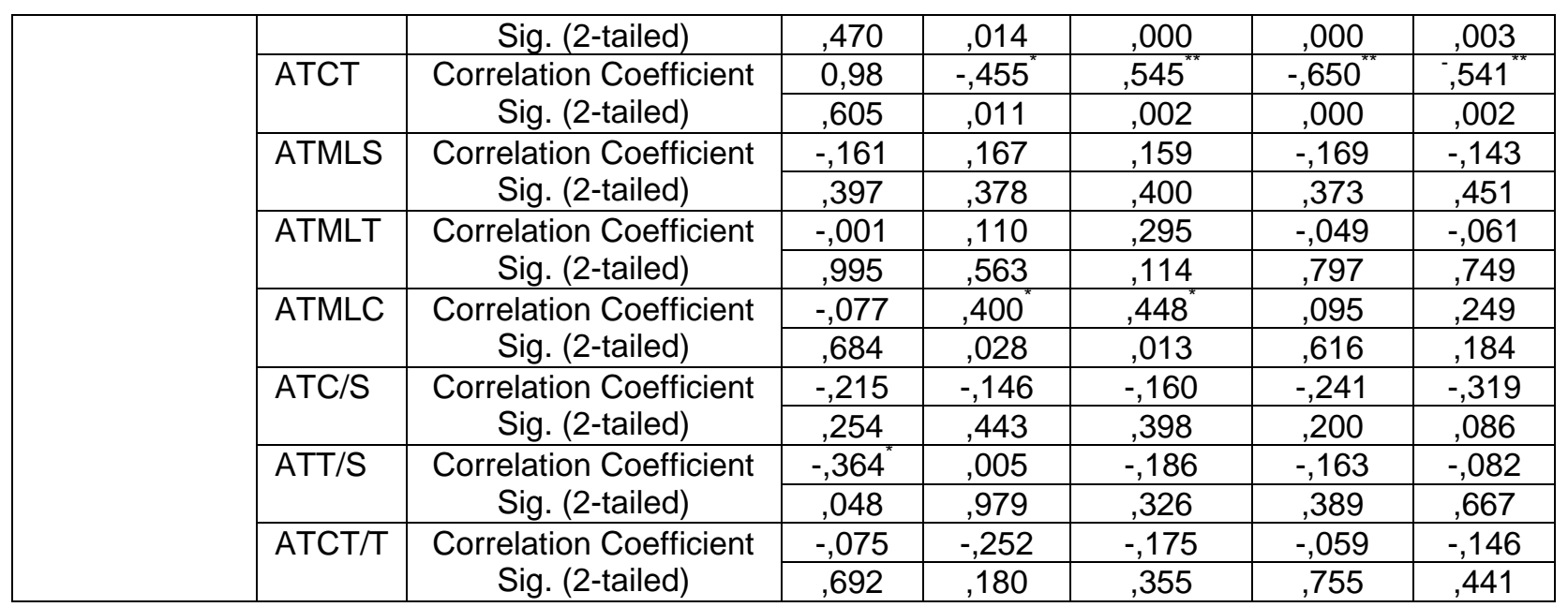

Table 5 presents the results of the Spearman correlation test used to evaluate the relationship between each index of lexical and syntactic complexity. Firstly, the relationship between word count and lexical density and lexical sophistication is not significant $(p>0,05)$. Since the number of the word increases, the number of the lexical words decreases compared to the grammatical words. Moreover, if the number of words increases, the number of sophisticated words also decreases. However, there is a strong positive correlation between word count and number of different words $(p<0,01)$. There is a negative significant correlation between word count and TTR and lexical word variation $(p<0,01)$. The same rationale applies in this case as well. When the number of words increases, the number of word types decreases compared to the whole number of words.

When analyzing sentences and lexical density and sophistication, there is not a significant correlation among them $(p>0,05)$. However, there is a strong correlation between sentences and number of different words again $(p<0,01)$. There is a negative correlation between sentences and TTR and there is not a significant correlation between sentences and lexical word variation.

As for clause index of syntactic complexity, there is not a significant correlation with lexical density and lexical sophistication $(p>0,05)$. There is a strong correlation with the number of different words $(p<0.01)$. Moreover, there is a strong negative correlation with TTR and lexical word variation. When it comes to T-unit, there are similar results again. The correlation between T-unit and lexical density and sophistication and lexical word variation is not significant $(p>0,05)$. The correlation between $T$-unit and number of different words is significant $(p<0,01)$.

For dependent clauses and complex T-units, there is not a significant correlation between these two indices and lexical density and lexical sophistication $(p>0.05)$. However, the correlation between these two indices of syntactic complexity and number of different words is strongly significant $(p<0,01)$. Moreover, there is a strong negative correlation between dependent clause and complex T-unit, and TTR and lexical word variation.

As for mean length of $\mathrm{T}$-Unit and mean length of sentence, clause per sentence, T-Unit per sentence, complex T-Unit ratio, there is not a significant correlation between any syntactic and lexical complexity indices $(p>0,05)$. As for mean length of clause, there is a significant correlation with lexical sophistication and number of different words $(p<0,05)$. This shows that the students used more sophisticated and advanced words at the clausal level rather than sentence level.

\section{DISCUSSION AND CONCLUSION}

The current research investigated the syntactic and lexical complexity level of the B1 students of English. In addition, the research aimed at finding out the relationship between the syntactic and lexical complexity. Moreover, the research was conducted to understand if the task types make a difference in the syntactic and lexical complexity of the spoken production of the students.

The current research backs up earlier research results regarding the syntactic and lexical complexity. The results of the research give a precise understanding for students and teachers of how task types influenced the second language speaking performance of the students. In the current research, it was found that the students use more vocabulary in the personal task compared to the descriptive and narrative tasks. The 
students formed more sentences in the descriptive task in terms of quantity but their sentences were shorter in the descriptive task compared to the personal and narrative tasks. It can be said that the quality and complexity of the sentences tend to be lower in the descriptive tasks. The longest and the most complicated sentences were formed in the personal task. Complex T-unit was performed in the personal task. In terms of syntactic complexity, the students performed their best performance in the personal task with a mean score of 16, 92. Their second best performance was in the descriptive task with a mean score of 14,83. The third one became the narrative task with a mean score of 14,56 . When it comes to lexical complexity, the students performed their best in the personal task regarding lexical density. Moreover, they were the most successful at the personal task again as for the lexical sophistication. Regarding the lexical variation, the students used the most different words in the personal task. Their type-token ratios were really close to each other in all tasks. In terms of lexical word variation, the descriptive task was the best one that the students performed. As for lexical complexity in general, the students performed their best performance in the personal task with a mean score of 11,82 . Their second best performance was in the descriptive task with a mean score of 10,51. The third one became the narrative task with a mean score of 10,44 in the lexical complexity. The personal task was the task in which the students formed their most complex sentences in terms of syntactic and lexical complexity. The descriptive task was the second best task in which the students were successful at forming complex sentences. Although the narrative task had close scores with the descriptive task, it became the last task in which the students formed the least syntactically and lexically complex sentence.

The results of the research showed that the students use more different vocabulary regarding the task type. These results are in line with the study of Préfontaine \& Kormos (2015). They also found out that the word ratio changes according to the task types like descriptive or narrative. Gan (2012) stated that task types have an impact on the production and performance of the second language learners. Our results are also in line with Gan's (2012) findings in this matter since the students performed differently in different task types.

As discussed before, Tavakoli \& Skehan (2005) mentioned the sequence of the images had an influence on the task performance. Task design is an important factor because it can lead to a better or poorer performance. In the narrative task, there were more items compared to the descriptive task. This +element of the task increases the task complexity and if the complexity of the task increases, the syntactic and lexical complexity of the spoken production decreases.

Likewise, Halleck's (1995) research investigated the effect of the task type on the syntactic complexity. Halleck found out that the different tasks have an effect on the performance. The tasks were narrative, descriptive and role-play and asking a question task. He found a difference between role-play tasks and other tasks. The learners performed better in the narrative and descriptive tasks rather than the role play task $(M=6,11)$. Like our results, Halleck found a really close mean score of 8,02 in narrative and descriptive tasks. In our research, the mean scores of descriptive and narrative task are 14,83 and 14,56.

Nippold (2009) conducted quite interesting research on the topic familiarity. The participants were classified into the groups regarding their chess knowledge such as masters and rookies. Masters performed better in terms of syntactic complexity. These results are somehow in line with our results. This shows that if the students have enough knowledge beforehand, they tend to form more syntactically complex sentences. In our research, the students were better at the personal task rather than the other tasks since they knew the knowledge about themselves beforehand and they could produce more complex sentences compared to the descriptive and narrative tasks which they saw in the test-taking for the first time.

In their study, Bayazidi et al. (2020) investigated the effect of the task types on syntactic and lexical complexity. The researchers used the three different tasks like argumentative, descriptive and narrative tasks. They analyzed diversity, density and sophistication. The results of this research are also consistent with our research and it showed that the task type had an impact on the lexical richness. In their study, the argumentative task had the highest score for diversity and density whereas the narrative task had the highest score for sophistication. In our study, there was not an argumentative task but there was a personal task, instead and the personal task had the highest score for density and sophistication.

Finally, the results of the current research present some different data as opposed to the studies by Foster (2001). In their study, they used three different tasks like personal, narrative and decision-making by analyzing the lexical complexity. They found out that narrative tasks generate the highest complexity. However, in our research, the personal tasks generate higher lexical complexity compared to the narrative task. In their study, there is not a consistent result. Every aspect of lexical complexity changes in every one of the tasks. They stated that the personal tasks were not consistent in terms of lexical diversity. However, the findings for the narrative and decision-making tasks are the opposite of that for lexical sophistication. The narrative tasks continued to produce lower diversity numbers than decision-making tasks. In our research, 
the personal task had the highest score in density and sophistication. Nonetheless, in lexical diversity, there were some inconsistent results. For number of different words, the personal task had the highest score. As for TTR, the narrative and descriptive tasks had the same highest score. Finally, for lexical word variation, the descriptive task had the highest score.

The results of the current research have some pedagogical implications for material development, curriculum design, second language testing and instruction in L2 classrooms. This research has implications for which spoken tasks should be conducted and tested in EFL courses since one of the goals of the research was to understand which kind of tasks the students perform their best. The results show that the students perform their best performance in the personal task, which means the students are more comfortable to produce more complex and complicated sentences if the topic is related to their own life and already familiar with them. Moreover, the students performed better in a simple descriptive task rather than a complex storytelling task.

The research finds out that the students could not develop their syntactic and lexical complexity together. Their lexical performance was not as good as their syntactic performance. These results show that the instructors should pay more attention to the vocabulary knowledge of their students. The students lack the word knowledge that their overall proficiency level requires.

For testing and assessment, the syntactic and lexical complexity indices enable the testing to be more objective rather than a holistic assessment of the instructors. Especially with the development of the webbased technological analysers, it is convenient to analyse the second language performance of the students. This research also shows a way to analyse performance with objective criteria.

The current research has some limitations. These limitations can be leading to future research studies.

The first limitation of the study is the number of the students. The number of the students who participated in the research was 30 . Due to the limited number, the results cannot be generalized to the other groups. Future research studies can be done with a bigger group of participants.

The second limitation is the skill that the research focused on. The research focused on only speaking skill. Future studies can focus on both speaking and writing at the same time.

The third limitation of the research is the proficiency level of the students. The students were all at the same level. Future research studies can be done with mixed and different levels of proficiency. Moreover, future research can investigate the syntactic and lexical complexity in terms of development. The students can be tracked during a whole year if they are preparatory students and their development of syntactic and lexical complexity can be observed.

The fourth limitation of the research is that all students but only one student were just monolingual. Future studies can be done with multilingual or bilingual students.

The fifth limitation of the research is that only one aspect of the CAF triad was investigated. Future studies can focus on accuracy and fluency besides complexity.

\section{REFERENCE LIST}

Bayazidi, A., Ansarin, A. A., \& Mohammadnia, Z. (2020). Lexical Complexity as a Function of Task Type and Proficiency Level in the Speech Monologs of Iranian EFL Learners. Journal of Modern Research in English Language Studies, 7(1), 29-44.

Crossley, S. A., \& McNamara, D. S. (2009). Computational assessment of lexical differences in L1 and L2 writing. Journal of Second Language Writing, 18(2), 119-135.

Foster, P. (2001). Rules And Routines: A Consideration of Their Role in the Task-based Language Production of Native and Non-Native Speakers. Researching Pedagogical Tasks: Second Language Learning, Teaching, and Testing. Ed. Martin Bygate, Peter Skehan, Merrill Swain. Harlow: Longman: 75-93.

Gan, Z. (2012). Complexity measures, task type, and analytic evaluations of speaking proficiency in a school-based assessment context. Language Assessment Quarterly, 9(2), 133-151.

Halleck, G. B. (1995). Assessing oral proficiency: A comparison of holistic and objective measures. The 
Modern Language Journal, 79(2), 223-234.

$\mathrm{Lu}, \mathrm{X}$. (2010). Automatic analysis of syntactic complexity in second language writing. International journal of corpus linguistics, 15(4), 474-496.

Lu, X. (2012). The relationship of lexical richness to the quality of ESL learners' oral narratives. The Modern Language Journal, 96(2), 190-208.

Nippold, M. A. (2009). School-age children talk about chess: Does knowledge drive syntactic complexity?. Journal of Speech, Language, and Hearing Research, 52 (1), 856-871.

Pallotti, G. (2009). CAF: Defining, refining and differentiating constructs. Applied linguistics, 30(4), 590-601.

Préfontaine, Y., \& Kormos, J. (2015). The relationship between task difficulty and second language fluency in French: A mixed methods approach. The Modern Language Journal, 99(1), 96-112.

Richards, J. C., \& Rodgers, T. S. (2014). Approaches and methods in language teaching. Cambridge university press.

Robinson, P. (1995). Task complexity and second language narrative discourse. Language learning, 45(1), $99-140$.

Skehan, P. (1998). A Cognitive Approach to Language Learning. Oxford: Oxford University Press.

Tavakoli, P \& Skehan, P. (2005). Planning, task structure, and performance testing. Planning and Task Performance in a Second Language. Ed. Rod Ellis. Amsterdam: John Benjamins:193-218.

Van den Branden, K. (2006). Introduction: Task-Based Language Teaching In a Nutshell. Task-Based Language Education: From Theory to Practice. Ed. Kris Van den Branden. Cambridge: Cambridge University Press: $1-16$. 